\title{
BIOCOMPATIBILITY OF TITANIUM IMPLANTS COATED WITH BIOCOMPOSITE IN A RAT MODEL OF FEMORAL FRACTURE
}

\author{
DANIEL OLTEAN-DAN ${ }^{a}$, PETRE T. FRANGOPOL ${ }^{\&}$, \\ REKA BALINT ${ }^{\mathrm{b},{ }^{*},}$, GHEORGHE TOMOAIA ${ }^{\mathrm{a}, \mathrm{c}}$, \\ AURORA MOCANU ${ }^{b}$, MARIA TOMOAIA-COTISEL ${ }^{b, c}$
}

\begin{abstract}
The biocompatibility of uncoated titanium, Ti, nails and coated with an innovative biocomposite is assessed in a rat model of femoral fracture. The biocomposite is based on multi-substituted hydroxyapatite, ms-HAP containing $\mathrm{Mg}, \mathrm{Zn}$ and $\mathrm{Si}$, and is used as a coating material deposited on $\mathrm{Ti}$ implants, due to the excellent biocompatibility and osteoconductive property of ms-HAP. Specific focus has been given to biocomposite coating made of ms-HAP functionalized with collagen (ms-HAP/COL, core/shell nanoparticles) NPs embedded into poly lactic acid, PLA, matrix and finally covered by COL layer. This coating on Ti implants is noted as ms-HAP/COL@PLA/COL and named HAPc, and is characterized by SEM images and EDX spectra. Twenty-four Wistar albino rats with left femoral fracture, were used and divided in two equal groups, namely the control group, CG, with uncoated $\mathrm{Ti}$ implants and HAPc rat group with $\mathrm{Ti}$ implants coated with HAPc biocomposite through layer-by-layer, LBL, dip coating technique. After two- and eight-weeks postsurgery, they were evaluated by bone markers serum concentration, micro-CT and histological study. The alkaline phosphatase (ALP) and osteocalcin (OCN) expression, in the HAPc group showed higher values with an osteoblastic activity more intense and a more advanced callus stage. The micro-CT results showed that biocomposite coating significantly increased the bone volume/ tissue volume (BV/TV). The most advanced stage of bone remodelling with osseointegration of the Ti implants was observed in HAPc group, where the new formed trabecular bone is almost completely replaced by compact bone.
\end{abstract}

a Iuliu Hatieganu University of Medicine and Pharmacy, Department of Orthopedics and Traumatology, 47 Gen. Traian Mosoiu Str., RO-400132, Cluj-Napoca, Romania

b Babeş-Bolyai University, Faculty of Chemistry and Chemical Engineering, Research Center of Physical Chemistry, 11 Arany Janos Str., RO-400028, Cluj-Napoca, Romania

c Academy of Romanian Scientists, 3 Ilfov Str., RO-050044, Bucharest, Romania

\& Deceased on December 11, 2020

* Corresponding author: Reka.Balint@ubbcluj.ro 
DANIEL OLTEAN-DAN, PETRE T. FRANGOPOL, REKA BALINT, GHEORGHE TOMOAIA, AURORA MOCANU, MARIA TOMOAIA-COTISEL

This study provides a novel strategy for the treatment of bone fracture with a superior osseointegration of biocomposite coated $\mathrm{Ti}$ implants and potential orthopaedic applications.

Keywords: biocompatibility, titanium implants, biocomposite coating, multisubstituted hydroxyapatite, PLA, collagen

\section{INTRODUCTION}

The development of novel orthopaedic and dental implants has been focused for many years to produce an optimal osseointegration between the implanted material and the living bone, without interposition of non-bone tissue [1]. To accomplish this major objective an optimal boneimplant interface needs to be designed and created through modification of the implant surface topography and chemical composition. The concept of direct contact between the bone and the implant was described as direct structural and functional connection between the living bone and the surface of a load-carrying implant [1-5] to prevent the fibrous tissue attachment.

An ideal implant material needs to have a biocompatible chemical composition similar to that of bone to avoid adverse bone tissue reaction, and to minimise bone resorption around the implant. Therefore, a newly developed implant needs to obey the requirements of biocompatibility and safety, and it must undergo rigorous testing in vivo. Results obtained in vitro studies might be difficult to extrapolate to the in vivo situation. For this purpose, the use of animal model is a crucial step in orthopaedic implants testing prior to clinical use in humans or in animals.

To achieve osseointegration various metals, ceramics and polymers were used. Major metal used was titanium, $\mathrm{Ti}$, and its alloys being recognized as biocompatible material to promote osseointegration $[4,6,7]$.

The host bone response to a $\mathrm{Ti}$ implant is influenced by its topography, roughness and porosity. It was observed that Ti surface morphology influences osteoblast and osteoclast attachment and metabolism [8]. However, the immune system is still considering $\mathrm{Ti}$ implants as foreign bodies and fibrosis around the implants can occur. In this case the osteoblasts are constrained to create bone ingrowth at the surface of the implant and osseointegration is restricted. This situation leads to loosening and failure of the implant $[9,10]$. The limitation of fibrous tissue production around the Ti implant can be reached by bioactive coating of the Ti surface with hydroxyapatite which can lead to benefits in terms of cell adhesion, differentiation, and bone matrix formation [9]. 
The hydroxyapatite can be substituted to create a composite more chemically closer to the normal bone tissue. Cationic substitution of the calcium ions within the hydroxyapatite, HAP, lattice can be performed with $\mathrm{Mg}^{2+}, \mathrm{Zn}^{2+}$, or $\mathrm{Ag}^{+}[10,11]$. Ionic substitution modifies the physico-chemical parameters of the hydroxyapatite, such as crystal morphology, degree of crystallinity, solubility and thermal stability, leading to various biological responses. Partial substitution, even in small quantities, leads to increased solubility and thermal stability of the hydroxyapatite lattice [11] and facilitates bone regenerative capacity, by promoting the cellular response. Silicon, magnesium and zinc increased bioactivity and implant integration and stimulated bone formation by facilitating the adhesion, proliferation and differentiation of osteoblasts [12]. Zinc substituted HAP and strontium substituted HAP were used in orthopaedic cement and it has been histologically shown that these materials were surrounded by bone tissue compared to poly methyl methacrylate, PMMA, which is encapsulated in fibrous tissue $[13,14]$. Collagen jointly with HAP have shown superior bioactivity and biocompatibility with better integration, reduced local reactions and earlier remodeling around the $\mathrm{Ti}$ rods, but they are rather fragile structures that can cause bone lysis [15].

The aim of the current study was to evaluate the biocompatibility and osseointegration of uncoated Ti nails [16-18] and coated Ti implants with biomimetic composite, in a rat model of femoral fracture. We selected this animal model [16] because most fractures occur in elderly patients with advanced osteoporosis of femur. Thus, we want to evaluate whether we can bring improvements in the osseointegration of $\mathrm{Ti}$ implants in the case of this population category at risk.

\section{RESULTS AND DISCUSSION}

\section{Surface morphology of HAPc biocomposite coated Ti implants}

FE-SEM image of grit-blasted and acid etched standard (control) Ti implant (Fig. 1A) and EDX spectrum of Ti implant shown in Fig. 1B, before and after coating by ms-HAP/COL@PLA/COL (Figs. 1C,D), which is made through $\mathrm{LBL}$ dip coating method on the surface of Ti implant.

As shown in Fig. 1C, the surface of the HAPc, ms-HAP/ COL@PLA/COL, biocomposite is rather porous with the COL fibers on its surface. The surface of coated Ti implants was also characterized by EDX spectrum (Fig. 1D), which gives the surface chemical composition of coating on $\mathrm{Ti}$ implant corresponding practically to ms-HAP (i.e., HAP-1.5\%Mg$0.2 \% \mathrm{Zn}-0.2 \% \mathrm{Si}$ ) composition. 
In the EDX spectrum, all constitutive elements: $\mathrm{Ca}, \mathrm{P}, \mathrm{O}, \mathrm{Mg}, \mathrm{Zn}$ and $\mathrm{Si}$ of ms-HAP are practically given. This finding indicates a homogeneous distribution of ms-HAP within HAPc biocomposite which contains also collagen and PLA.

In EDX spectrum, Au can be seen, which was used for enhancing FESEM imaging. The topography of outermost COL layer was visualized by AFM images showing the nanofibers of collagen on the surface of HAPc coating on $\mathrm{Ti}$ implants (not shown).

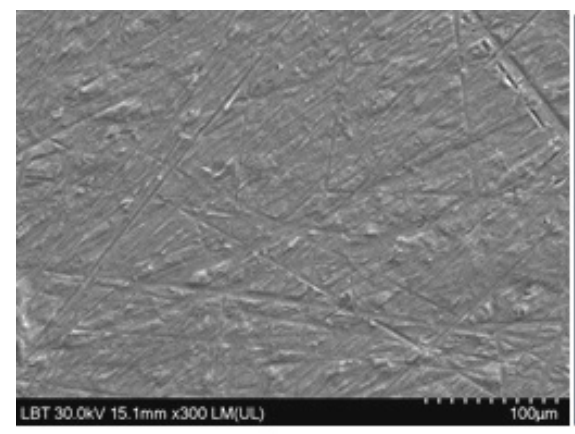

A

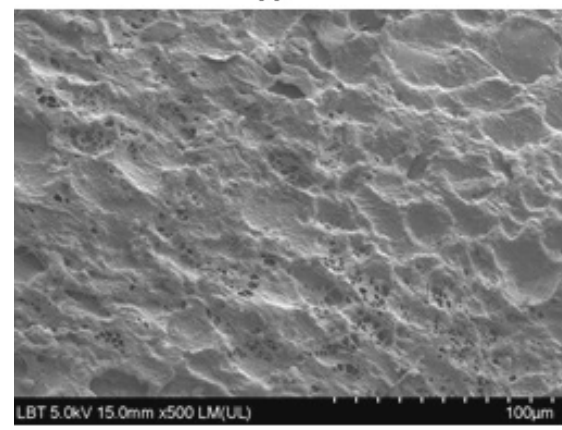

$\mathrm{C}$

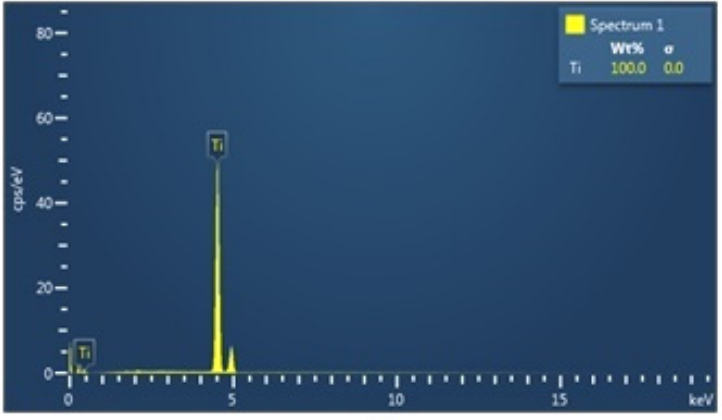

B

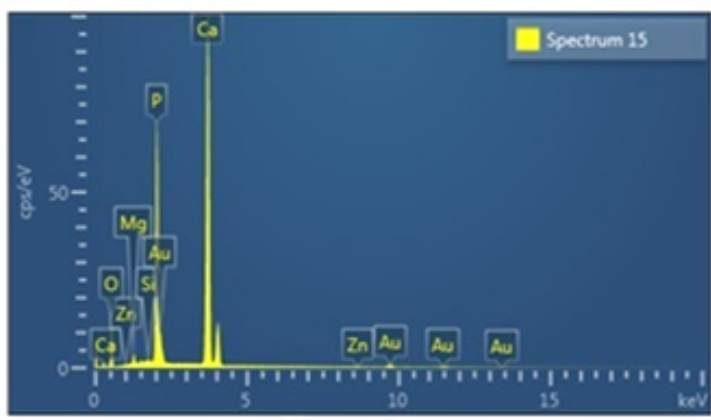

D

Figure 1. A) FE-SEM image on the surface microstructure of uncoated Ti rod (20 $\mathrm{mm} \times$ diameter $1 \mathrm{~mm}$ ) and its EDX spectrum in Fig. 1B, showing the highest purity of Ti rod, and after its coating with HAPc biocomposite, SEM image (Fig. 1C) and EDX spectrum (Fig. 1D), indicating a porous HAPc structure, multi doped with essential elements.

\section{Bone markers: alkaline phosphatase and osteocalcin results}

An increase in alkaline phosphatase (ALP) concentration (Fig. 2A) was observed in both groups at 2 weeks compared to initial values $(p<0.0001)$. The most significant increase was in the HAPc group (173\%), compared to CG $(152 \%), p<0.001$. At 8 weeks, ALP values decrease $(p<0.0001)$, with approximately equal or even lower bone serum concentrations, compared to the initial values ( 0 weeks). 
The osteocalcin (OCN) values (Fig. $2 \mathrm{~B}$ ) increased substantially in both groups, compared to initial values, with the highest increase of $230 \%$ in the HAPc group. Furthermore, values in the control group, CG, at two weeks were significantly lower compared to the HAPc group $(p<0.0001)$. Afterwards, at eight weeks, OCN expression decreased significantly, remaining with $31 \%-36 \%$ higher than the initial value $(p<0.05)$.

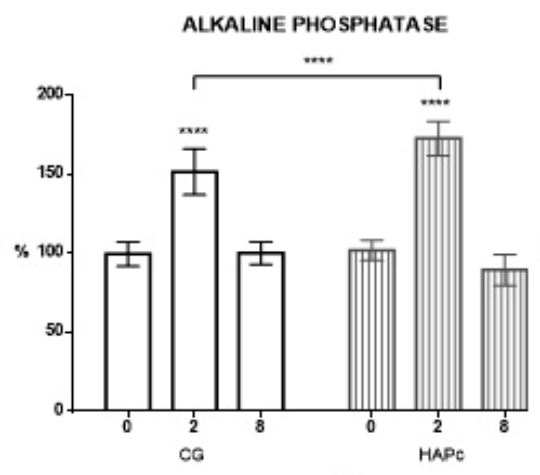

A

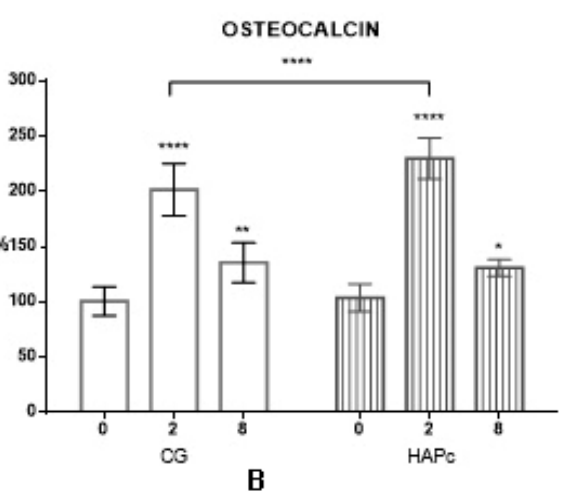

B

Figure 2. Bone markers, alkaline phosphatase $(A)$ and osteocalcin (B), serum concentration at zero time (initially), two- and eight-weeks post-surgery; *statistically significant $p<0.05 ;$ ** statistically significant $p<0.01$; ${ }^{* * *}$ statistically significant $p<0.001 ;{ }^{* * * *}$ statistically significant $p<0.0001$.

Table 1. Bone markers, alkaline phosphatase (ALP) and osteocalcin (OCN), serum concentration at initial ( 0 weeks), two- and eight-weeks post-operatively; ${ }^{*}$ statistically significant $p<0.05$; ** statistically significant $p<0.01$; ${ }^{* * *}$ statistically significant $p<0.001 ;{ }^{* * * *}$ statistically significant $p<0.0001$.

\begin{tabular}{|l|l|l|l|}
\hline \multicolumn{2}{|l|}{ Rat group } & CG & HAPC \\
\hline ALP & 0 weeks & $100 \pm 13$ & $102 \pm 6$ \\
$(\%)$ & 2 weeks & $152 \pm 14^{* * *}$ & $173 \pm 10^{* * * *}$ \\
& 8 weeks & $100 \pm 7$ & $89 \pm 8$ \\
\hline OCN & 0 weeks & $100 \pm 15$ & $104 \pm 13$ \\
$(\%)$ & 2 weeks & $202 \pm 24^{* * * *}$ & $230 \pm 18^{* * * *}$ \\
& 8 weeks & $136 \pm 18^{* *}$ & $131 \pm 8^{*}$ \\
\hline
\end{tabular}

\section{Micro-CT results}

Table 2. Implant osseointegration assessed by micro-CT; bone volume per total tissue volume (BV/TV) and the mean trabecular number (Tb.N); * $p$ < 0.05: HAPc group vs CG.

\begin{tabular}{|l|c|c|}
\hline Rat group & CG & HAPc \\
\hline BV/TV $(\%)$ & $25.5 \pm 4.3$ & $38.8 \pm 5.4^{*}$ \\
\hline Tb.N $(1 / \mathrm{mm})$ & $154 \pm 18$ & $180 \pm 18^{*}$ \\
\hline
\end{tabular}


At eight weeks after implantation, the development of newly formed bone and the degree of osseointegration were evaluated by the formation of trabecular and cortical bone at the fracture site (Figs. 3A,B).

An in vivo evaluation of biocompatibility of the two different surfaces of uncoated $\mathrm{Ti}$ and coated HAPcTi implants is required for the understanding of the role of surface features and chemical composition of coatings on $\mathrm{Ti}$ implant surface in the osseointegration of implants and living host bone.

\section{Histological results}

The biocompatibility of uncoated and coated $\mathrm{Ti}$ implants and their osseointegration at the fracture level were assessed by using optical microscopy, on hematoxylin and eosin (H\&E) stained tissue samples from each of the two studied animal groups, at eight-weeks postoperatively.
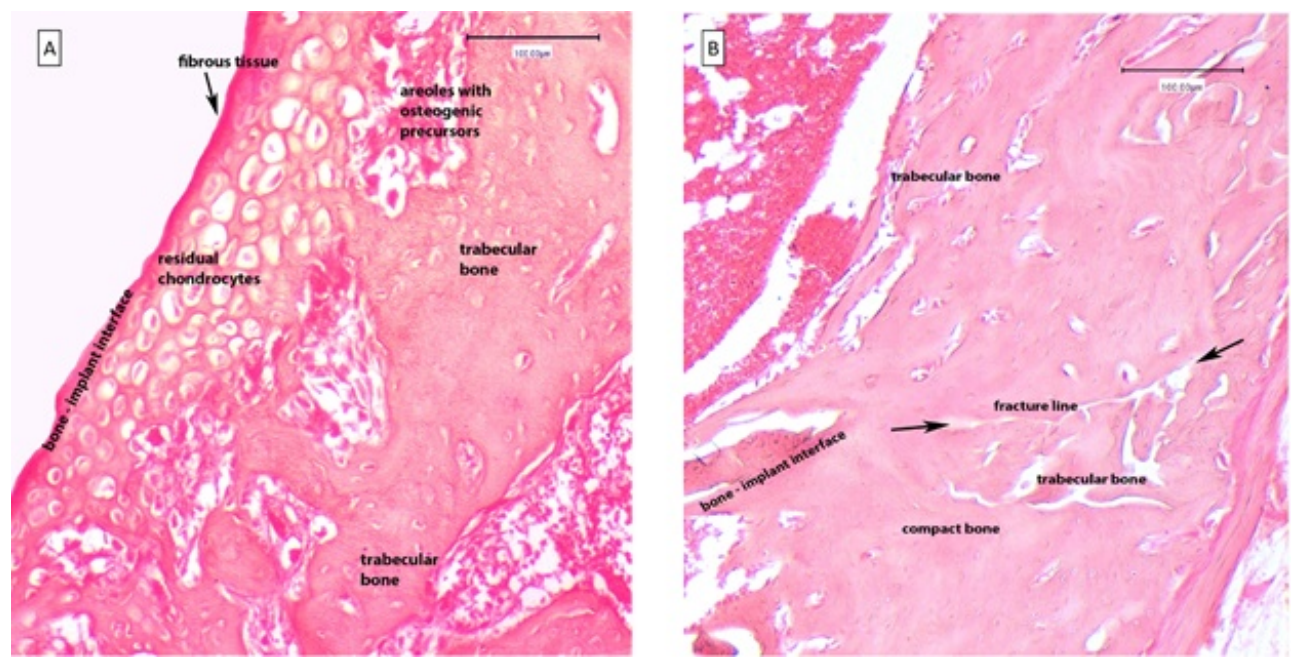

Figure 3. Optical microscopy images of H\&E stained slides of tissue samples at bone-implant interface near the fracture site, at eight weeks after implantation. (A): control group revealed fibrous tissue in the proximity of Ti intramedullary implant and residual cartilaginous tissue, indicating a transition from cartilaginous precursors to incipient bone trabeculae formation. (B): HAPc group displayed around HAPcTi implants, the bone trabeculae well defined, compact bone with lamellar disposition of bone matrix, and osteocytes around Havers canals, with osteoblasts lining their surface, and clear delimitation of areole between the trabeculae, with areas of compact lamellar bone deposition. 


\section{Results Analysis}

Fig. 1A shows the FE-SEM image of the uncoated Ti implant, which was grit-blasted and acid etched treated, having the highest purity (Fig. 1B). Fig. $1 \mathrm{C}$ displays the FE-SEM image and the surface structure of Ti implant coated with HAPc composite. The morphology of the composite coating, ms-HAP/COL@PLA/COL, illustrates that the surface of HAPc composite is porous, and NPs of ms-HAP/COL are well dispersed in PLA matrix. The EDX spectrum (Fig. 1D) unveils the presence of ms-HAP as HAP-1.5\%Mg$0.2 \% \mathrm{Zn}-0.2 \% \mathrm{Si}$ homogeneously distributed within HAPc composite.

The alkaline phosphatase (Fig. 2A) and osteocalcin (Fig. 2B) play a vital role in bone mineralization being valuable biochemical markers in assessing newly bone formation. Osteoblasts, the bone-forming cells, express ALP on their surface, while ALP isoforms can be derived from bone or nonspecific tissue. Osteocalcin is the most abundant non-collagenous bone protein [19].

In this work, an experimental investigation on an animal (rat) model was achieved using diaphyseal femoral fracture. To stabilize the fracture site, titanium intramedullary nails uncoated or coated with HAPc composite were introduced in a retrograde method. Afterwards, the rats were divided into two distinct groups of twelve rats each; namely group CG: the control group where uncoated titanium nails were implanted; HAPc group: where titanium nails coated with ms-HAP/COL@PLA/COL composite were implanted. Ionic substitution within the HAP lattice, specifically $1.5 \% \mathrm{Mg}, 0.2 \% \mathrm{Zn}$ and $0.2 \% \mathrm{Si}$, causes changes in its surface structure and, in terms of its electrical charge, increases the solubility and capacity of substituted HAP to participate in the natural process of bone remodeling, in substantial agreement with related materials [20].

The HAP/COL composites have been used in rabbits to demonstrate their ability to fulfil osteochondral defects, showing the formation of active bone tissue [21-23]. The HAP/COL composite was also used to cover the titanium nails placed subperiosteally at the level of the skull, which were completely surrounded by bone tissue, while more than $50 \%$ of the titanium rods coated with simple HAP were encapsulated in fibrous tissue [24]. In addition, collagen coating on titanium enhances the connection between titanium and newly formed bone and can be used in cases that require better osseointegration [25].

The use of titanium coated with calcined hydroxyapatite-collagen ensures both the mechanical stability and the rigidity of the fixation method, maintaining long-term osteoconductive properties [26]. In addition, substituted HAP continuously releases its ions, with specific effects in bone biochemistry and mineral homeostasis [27]. 
Also, the use of titanium coated with type I collagen/chondroitin sulphate showed a better integration at the bone-implant interface in case of osteoporosis [28].

Poly lactic acid is a bioresorbable polyesters [29], but its clinical use is limited due to low hydrophilicity and difficulty in controlling the rate of hydrolysis [30]. Therefore, the use of HAP and PLA composites can reduce the rate of PLA degradation and strengthen the biomaterials [31]. Experimental studies [32-35] on bone tissue models have shown that these composites have an active role in bone formation, by facilitating ossification without producing inflammation [34].

Titanium surfaces coated with silicon [36], zinc [37] and magnesium [38] substituted hydroxyapatite enhance the bioactivity and promote osteogenic differentiation of preosteoblasts in vitro, which has a real potential to enhance implants osseointegration.

The ALP evolution in the HAPc group shows a faster growth compared to the control group until two weeks, after which the ALP activity decreases [39].

Our results revealed a significant increase of alkaline phosphatase in animals treated with HAPc coated titanium nails in the bone consolidation process with a $73 \%$ increase significantly over the initial values. Moreover, in vitro studies, used to examine the osteoblastic differentiation of cells cultured on days 7,14 and 21, showed the maximum ALP activities at 14 days of culture and decreased thereafter in all groups and samples. This is a typical dynamic of the ALP activity, characteristic of early osteoblastic differentiation [40]. ALP increases the local concentration of phosphate ions and the deposition of calcium salts. Moreover, osteoblasts actively secreted bone matrix and released large amounts of alkaline phosphatase. This activity is correlated with the formation of new bone [41].

Hydroxyapatite and especially multi-substituted hydroxyapatite create a microclimate favourable to cellular recruitment, and due to its osteoconductive capacity, stimulates the activity of osteoblasts and facilitates bone strengthening, demonstrated by a higher activity of ALP. Subsequently, after $2^{\text {nd }}$ to $8^{\text {th }}$ week, ALP activity reverted to approximately the initial values. The ALP values at eight weeks were significantly lower than initial values (at 0 weeks) in groups where titanium intramedullary nails coated with multisubstituted hydroxyapatite were used. The marked decrease in ALP activity indicates an increase in fracture focal stability and a more advance stage of bone consolidation, which is more evident in these groups.

Osteocalcin has been shown to be a more specific marker in changes in bone metabolism than alkaline phosphatase, being a late marker of bone formation [42]. Alkaline phosphatase, although less specific, is an effective marker in increased bone turnover in fracture healing, but its elevated levels are maintained for a lower period of time than osteocalcin. According ALP activity at 2 weeks, the OCN values increased significantly 
in both groups compared to initial values with the highest increase in the HAPc group. Furthermore, values in the control group at two weeks were significantly lower compared to the HAPc group. At eight weeks, OCN expression decreased significantly in all groups compared to values at two weeks, with $31 \%-36 \%$ higher than initial values with statistical differences for all groups $(p<0.05)$.

Normally, levels of OCN changes are evident in the final stages of osteogenic differentiation, when this protein is secreted by osteoblasts. Osteocalcin activates both osteoblasts and osteoclasts during early bone formation and causes an earlier onset of bone remodelling process. This has been demonstrated in vitro and in vivo studies on Wistar albino rats in which cylindrical nanocrystalline hydroxyapatite/collagen implants have been implanted in the tibia by accelerating bone formation and regeneration [43].

Consequently, in terms of the dynamics of ALP activity and OCN expression, in the groups treated with multi-substituted hydroxyapatitecollagen-PLA coated titanium intramedullary nails, the values are higher, with osteoblastic activity more intense and bigger callus. Considering that alkaline phosphatase is an early bone formation marker, at eight weeks its activity diminished, when the fracture is stabilized by the newly formed trabecular bone. On the other hand, OCN as a late bone formation marker, its values remained higher even after eight weeks.

Micro-CT assessments and histological evaluations were employed to investigate the osseointegration and bone formation around the implants and the host bone around them. Bone volume relative to total tissue volume (BV/TV) and trabecular number (Table 2) provide the changes, regarding the bone neo-formation between the intramedullary nail and the host bone around it [44].

Furthermore, hematoxylin and eosin stains showed the changes in the activity of cells and tissues and in the degree of mineralization at the bone-implants interface. Regarding the quantitative results of implant osseointegration assessed by micro-CT, coating composed of ms-HAP/ COL@PLA/COL composite produced anabolic effect on bone around the implant, with significantly increased of BV/TV and Tb.N compared to control group. In the case of ovariectomized rats, good results have been demonstrated regarding osteointegration, in the case of strontium hydroxyapatite coatings [45].

H\&E colored slides analysis, at eight weeks post fracture, revealed bone remodeling processes at fracture site and displayed major differences between the investigated groups. In control group (Fig. 3A), there was an intense eosinophilic band representing fibrous tissue at bone-implant interface, which indicates a lower osseointegration capacity of titanium nails in this group, while HAPc group implants (Fig. 3B) revealed a better osseointegration. 
The novelty of our study is the use of a new biomimetic composite, consisting of multi-substituted hydroxyapatite with silicon, magnesium and zinc, NPs covered with collagen and jointly incorporated in a poly lactic matrix in a unilateral femoral fracture rat model. We also evaluated the effect of those coatings at different time points ( 0 weeks, 2 weeks and 8 weeks postsurgery) during the bone consolidation and osseointegration process. In all the cases, we used biocomposite coatings and we didn't encounter any case with inflammation signs.

Our results showed that more bone was formed during the first two weeks in the fracture focal point, especially in the rats treated with HAPc coating. Regarding the bone implant contact in the metaphyseal region, the coating based on multi-substituted hydroxyapatite showed better results compared to the control group at 8 weeks postoperatively. Micro-CT and histological changes revealed that HAPc coating of the conventional titanium nails enhances bone healing and osseointegration of the intramedullary implants, as well. In clinical situation, these findings can play an important role by diminishing the risk of cut-outs of the implant and also in the prevention of the implant loosening.

The HAPc coating, comprising Mg-Zn-Si-HAP and COL in PLA matrix, is enriched in $\mathrm{Mg}$ and is self-assembled on Ti implants. This HAPc coating can induce in vivo early formation of a bone-to-implant interface in good agreement with recent studies on related materials [46-52], including the bone sites with osteoporosis, making this surface treatment of the highest osteogenic potential, due to the release of $\mathrm{Mg}, \mathrm{Zn}$, and $\mathrm{Si}$ in vivo.

\section{CONCLUSIONS}

Titanium intramedullary nails coated with multi-substituted hydroxyapatite and collagen in a poly lactic acid matrix enhance bone healing and also increase implant osseointegration by improving the bone microstructure around the implant. In the case of clinical applications, these implants may decrease the risk of implant loosening.

\section{EXPERIMENTAL SECTION}

\section{Materials, sample preparation and characterization}

Rods of Ti (99.6\% purity) were purchased from Goodfellow Cambridge Limited, Huntingdon, England. The Ti implants were prepared, as follows: the surface of Ti rods $(20 \mathrm{~mm} \times$ diameter $1 \mathrm{~mm}$ ) was firstly improved by gritblasting with $\mathrm{P} 500$, and cleaned by ultrasonication process using a ultrasonic processor Sonics Vibra-Cell, model VCX 750 (Sonics \& Material Inc., Newtown, 
CT, USA) in deionized water at room temperature for 2 hrs. Subsequently, the Ti rods were chemically activated by acid etching with $50 \mathrm{wt} \%$ phosphoric acid solution, for $10 \mathrm{~min}$, and sterilized through ethylene oxide procedure leading to standard Ti implants, noted Ti implants, of controlled surface roughness. Then, Ti implants were further coated by HAPc composite, deposited on $\mathrm{Ti}$ rods through layer-by-layer, LBL, method resulting HAPc Ti coated implants. The HAPc composite comprises multi-substituted hydroxyapatite, ms-HAP: HAP- $1.5 \% \mathrm{Mg}-0.2 \% \mathrm{Zn}-0.2 \% \mathrm{Si}$, nanoparticles (NPs) covered by collagen, COL, namely ms-HAP/6\%COL (core/shell NPs), incorporated into poly lactic acid, PLA, matrix (ms-HAP/COL@PLA) and finally covered by COL layer (ms-HAP/ COL@PLA/COL), which was deposited on Ti surface through self-assemblies (LBL) method.

Characterization of HAPc composite coatings on $\mathrm{Ti}$ implants was carried out by field emission scanning electron microscope (FE-SEM), Hitachi SU-8230, to explore the structure of composite surface. FE-SEM was equipped with Oxford energy-dispersive X-ray spectrometer for elemental analysis (energy-dispersive X-ray, EDX, spectra). Therefore, FE-SEM and EDX were used for surface morphology and elemental analysis of coated Ti implants.

\section{Study protocol}

The research was carried out in accordance with the principles of the Basel Declaration and recommendation of ARRIVE guidelines. The animal research protocol was approved by Veterinary Sanitary Committee of Cluj County, Romania (Approval No. 85/19.07.2017) and Ethics Committee of "Iuliu Hatieganu" University of Medicine and Pharmacy, Cluj-Napoca.

In the present study, Wistar albino rats $(\mathrm{N}=24)$ were used, and divided in two equal groups ( $N=12 /$ group): $C G$ control group, with uncoated $\mathrm{Ti}$ implants and HAPc group with Ti implants coated with HAPc coating, noted HAPcTi implants.

\section{Surgical procedures}

The rats were two months old, weighing $218 \pm 11 \mathrm{~g}$. They were anesthetized with an intramuscular cocktail of $2 \%$ xylazine and $10 \%$ ketamine. The surgeries were performed by a team of two orthopedic surgeons. After the establishment of the surgical asepsis through a lateral approach of the thigh, a transverse fracture of the femoral diaphysis was performed.

By a longitudinal incision at the knee level, uncoated Ti implants (CG, N=12) and coated with HAPc: ms-HAP/COL@PLA/COL (HAPc group, $\mathrm{N}=12$ ) were introduced in the left femur. At the end, the subcutaneous plane and the tegument were sutured. Postoperatively, the animals were kept in cages, in a controlled environment with a day/night cycle of $12 \mathrm{~h}$, without food restrictions, at a temperature of $22^{\circ} \mathrm{C}$. 
Animal euthanasia was performed by overdose of anesthetic at 2 weeks $(\mathrm{N}=12, \mathrm{~N}=6 / \mathrm{group})$ and at 8 weeks $(\mathrm{N}=12, \mathrm{~N}=6$ /group), and the left femoral bone was carefully taken, by push-out method, not to destroy bone callus and placed in $10 \%$ formaldehyde.

\section{Alkaline phosphatase and osteocalcin}

Bone formation markers, osteocalcin (OCN) and alkaline phosphatase (ALP), were evaluated using the non-specific ELISA kit ER1205, Rat OC / BGP (Osteocalcin), commercially available (Wuhan Fine Biological Technology Co) and OSR6504 reagent (alkaline phosphatase), for use on the AU 680 system (Beckman Coulter, USA). Around $0.6 \mathrm{~mL}$ of blood was harvested from each rat, at the beginning of the experiment $(\mathrm{N}=24)$, at two weeks $(\mathrm{N}=24)$ and at 8 weeks $(\mathrm{N}=12)$.

\section{Micro-CT}

Multilevel threshold was applied to discriminate the bone and calcified cartilage, dense cortical bone and un-mineralized tissue. At the time of sacrifice, the left femoral bone with implants ( $N=12 /$ group) were scanned using Bruker micro-CT system SkyScan 1172 (Kontich, Belgium). The volume of interest (VOI) was established at $2 \mathrm{~mm}$ below the growth plate, with a height of $1 \mathrm{~mm}$ and a ring of $1.5 \mathrm{~mm}$ diameter around the implant. Afterwards, the bone volume percent (BV/TV) and the mean trabecular number (Tb.N) were assessed within the volume of interest.

\section{Histological assessment}

The biocompatibility of uncoated and coated $\mathrm{Ti}$ implants and their osseointegration at the fracture level was assessed by using optical microscopy, on hematoxylin and eosin (H\&E) stained tissue samples from each of the two studied animal groups, at two- and eight-weeks postoperatively. After harvesting, the rat femurs were fixed in $4 \%$ paraformaldehyde solution for the first $24 \mathrm{hrs}$. Then, $5 \%$ nitric acid solution, continuously shacked and changed daily for 10 days, was used in order to get the decalcification of bone samples. Samples were then dehydrated in increasing degrees of alcohol $(50 \%, 75 \%, 100 \%)$, cleared in xylene (for alcohol removal) and embedded in paraffin. Afterwards, the cross sections of $5 \mu \mathrm{m}$ thickness attached on glass slide were deparaffinised, rehydrated and then stained with $\mathrm{H} \& \mathrm{E}$. The slides were photographed by using a Leica DMD 120 microscope. At two weeks after implantation, the inflammatory response was not identified. At eight weeks postoperative, the formation of newly formed bone and the degree of osseointegration were assessed by the formation of trabecular and cortical bone at fracture site $[17,18]$. 
BIOCOMPATIBILITY OF TITANIUM IMPLANTS COATED WITH BIOCOMPOSITE IN A RAT MODEL OF FEMORAL FRACTURE

\section{Statistical analysis}

For statistical analysis GraphPad Prism 6 for Windows was used. Values of bone markers concentration were converted to percentages and were defined as the mean value \pm standard deviation (SD). Statistical significance was identified using one-way ANOVA test followed by Tukey post-hoc test.

\section{ACKNOWLEDGMENTS}

This work was supported by grants of the Ministry of Research, Innovation and Digitization, CNCS/CCCDI-UEFISCDI, project number 186 and 481, within PNCDI III.

\section{REFERENCES}

1. A. F. Mavrogenis; R. Dimitriou; J. Parvizi; G. C. Babis; J. Musculoskelet. Neuronal Interact., 2009, 9(2), 61-71.

2. P. I. Branemark; Scand. J. Clin. Lab. Invest., 1959, 11(Suppl. 38), 1-82.

3. P. I. Branemark; J. Prosthet. Dent., 1983, 50(3), 399-410.

4. B. M. Isaacson; S. Jeyapalina; Orthop. Res. Rev., 2014, 6, 55-65.

5. R. D. Bloebaum; K. N. Bachus; N. G. Momberger; A. A. Hofmann; J. Biomed. Mater. Res., 1994, 28(5), 537-544.

6. R. Depprich; H. Zipprich; M. Ommerborn; C. Naujoks; H. P. Wiesmann; S. Kiattavorncharoen; H. C. Lauer; U. Meyer; N. R. Kubler; J. Handschel; Head Face Med., 2008, 4, 30. DOI: 10.1186/1746-160X-4-30.

7. P. Thomsen; C. Larsson; L. E. Ericson; L. Sennerby; J. Lausmaa; B. Kasemo; J. Mater. Sci. Mater. Med., 1997, 8(11), 653-665.

8. A. B. Novaes Jr; S. L. Scombatti de Souza; R. R. Martins de Barros; K. K. Y. Pereira; G. lezzi; A. Piattelli; Braz. Dent. J., 2010, 21(6), 471-481.

9. W. Wang; C. K. Poh; Titanium Alloys in Orthopaedics. In Titanium Alloys Advances in Properties Control, J. Sieniawski, W. Ziaja Eds.; Intech Open; London, UK, 2013, chapter 1, pp. 1-20.

10. A. Mocanu; G. Furtos; S. Rapuntean; O. Horovitz; C. Flore; C. Garbo; A. Danisteanu; G. Rapuntean; C. Prejmerean; M. Tomoaia-Cotisel; Appl. Surf. Sci., 2014, 298, 225-235.

11. F. Goga; E. Forizs; A. Avram; A. Rotaru; A. Lucian; I. Petean; A. Mocanu; M. Tomoaia-Cotisel; Rev. Chim. (Bucharest), 2017, 68(6), 1193-1200.

12. J. T. B. Ratnayake; M. Mucalo; G. J. Dias; J. Biomed. Mater. Res. B Appl. Biomater., 2017, 105(5), 1285-1299.

13. D. Shepherd; S. M. Best; Biomed. Mater., 2013, 8(2), 025003. DOI: $10.1088 / 1748-6041 / 8 / 2 / 025003$ 
DANIEL OLTEAN-DAN, PETRE T. FRANGOPOL, REKA BALINT, GHEORGHE TOMOAIA, AURORA MOCANU, MARIA TOMOAIA-COTISEL

14. N. Kourkoumelis; Ann. Transl. Med., 2016, 4(Suppl. 1), S10. doi: 10.21037/atm.2016.10.03

15. H. C. Wu; T. W. Wang; J. S. Sun; Y. H. Lee; M. H. Shen; Z. R. Tsai; C. Y. Chen; H. C. Hsu; Materials (Basel), 2016, 9(3), 198. DOI: 10.3390/ma9030198

16. D. Oltean-Dan; G. B. Dogaru; M. Tomoaia-Cotisel; D. Apostu; A. Mester; H. R. Benea; M. G. Paiusan; E. M. Jianu; A. Mocanu; R. Balint; C. O. Popa; C. Berce; G. I. Bodizs; A. M. Toader; Gh. Tomoaia; Int. J. Nanomed., 2019, 14, 5799-5816.

17. R. Marsell; T. A. Einhorn; Injury, 2011, 42(6), 551-555.

18. A. Schindeler; M. M. McDonald; P. Bokko; D. G. Little; Semin. Cell. Dev. Biol., 2008, 19(5), 459-466.

19. Y. T. Tsao; Y. J. Huang; H. H. Wu; Y. A. Liu; Y. S. Liu; O. K. Lee; Int. J. Mol. Sci., 2017, 18(1), 159. DOI: 10.3390/ijms18010159.

20. D. M. Ibrahim; A. A. Mostafa; S. I. Korowash; Chem. Cent. J., 2011, 5(1), 74. doi: 10.1186/1752-153X-5-74

21. F. G. Lyons; J. P. Gleeson; S. Partap; K. Coghlan; F. J. O'Brien; Clin. Orthop. Relat. Res., 2014, 472(4), 1318-1328.

22. F. Yu; M. Li; Z. Yuan; F. Rao; X. Fang; B. Jiang; Y. Wen; P. Zhang; Int. J. Nanomed., 2018, 13, 7845-7858.

23. A. Tsuchiya; S. Sotome; Y. Asou; M. Kikuchi; Y. Koyama; T. Ogawa; J. Tanaka; K. Shinomiya; J. Med. Dent. Sci., 2008, 55(1), 91-99.

24. M. Uezono; K. Takakuda; M. Kikuchi; S. Suzuki; K. Moriyama; J. Biomed. Mater. Res. B Appl. Biomater., 2013, 101B(6), 1031-1038.

25. A. Murakami; T. Arimoto; D. Suzuki; M. Iwai-Yoshida; F. Otsuka; Y. Shibata; T. Igarashi; R. Kamijo; T. Miyazaki; Nanomedicine, 2012, 8(3), 374-382.

26. G. G. Walmsley; A. McArdle; R. Tevlin; A. Momeni; D. Atashroo; M. S. Hu; A. H. Feroze; V. W. Wong; P. H. Lorenz; M. T. Longaker; D. C. Wan; Nanomedicine, 2015, 11(5), 1253-1263.

27. D. Govindaraj; M. Rajan; M. A. Munusamy; A. A. Alarfaj; K. K. Sadasivuni; S. S. Kumar; Nanomedicine, 2017, 13(8), 2661-2669.

28. L. Kyllönen; M. D'Este; M. Alini; D. Eglin; Acta Biomater., 2015, 11, 412-434.

29. J. Li; X. L. Lu; Y. F. Zheng; Appl. Surf. Sci., 2008, 255(2), 494-497.

30. W. Yang; G. Yin; D. Zhou; J. Gu; Y. Li; H. Zhang; J. Mater. Sci. Technol., 2010, 26(8), 754-758.

31. P. Vashisth; J. R. Bellare; Nanomedicine, 2018, 14(4), 1325-1336.

32. Gh. Tomoaia; L. B. Pop; I. Petean; M. Tomoaia-Cotisel; Mater. Plast., 2012, 49(1), 48-54.

33. Y. Shapovalova; D. Lytkina; L. Rasskazova; A. Gudima; V. Ryabov; A. Filimoshkin; I. Kurzina; J. Kzhyshkowska; Eur. J. Cancer. Suppl., 2015, 13(1), 49-50.

34. P. Kubasiewicz-Ross; J. Hadzik; J. Seeliger; K. Kozak; K. Jurczyszyn; H. Gerber; M. Dominiak; C. Kunert-Keil; Ann. Anat., 2017, 213, 83-90.

35. Gh. Tomoaia; M. Tomoaia-Cotisel; A. Mocanu; O. Horovitz; L. D. Bobos; M. Crisan; I. Petean; J. Optoelectron. Adv. Mater., 2008, 10(4), 961-964. 
36. M. Honda; K. Kikushima; Y. Kawanobe; T. Konishi; M. Mizumoto; M. Aizawa; J. Mater. Sci. Mater. Med., 2012, 23(12), 2923-2932.

37. F. Yang; W. J. Dong; F. M. He; X. X. Wang; S. F. Zhao; G. L. Yang; Oral Surg. Oral Med. Oral Pathol. Oral Radiol., 2012, 113(3), 313-318.

38. S. F. Zhao; Q. H. Jiang; S. Peel; X. X. Wang; F. M. He; Clin. Oral Implants Res., 2013, 24(Suppl. A100), 34-41. doi.org/10.1111/j.1600-0501.2011.02362.x

39. R. Marom; I. Shur; R. Solomon; D. Benayahu; J. Cell. Physiol., 2005, 202(1), $41-48$.

40. D. H. Yang; D. W. Lee; Y. D. Kwon; H. J. Kim; H. J. Chun; J. W. Jang; G. Khang; J. Tissue Eng. Regen. Med., 2015, 9(9), 1067-1077.

41. I. Manjubala; M. Sivakumar; R. V. Sureshkumar; T. P. Sastry; J. Biomed. Mater. Res., 2002, 63(2), 200-208.

42. S. Shetty; N. Kapoor; J. D. Bondu; N. Thomas; T. V. Paul; Indian J. Endocrinol. Metab., 2016, 20(6), 846-852.

43. S. Rammelt; M. Neumann; U. Hanisch; A. Reinstorf; W. Pompe; H. Zwipp; A. Biewener; J. Biomed. Mater. Res. A, 2005, 73(3), 284-294.

44. J. Y. C. Choi; C. A. Choi; I. S. L. Yeo; J. Periodontal Implant Sci., 2018, 48(4), 202-212.

45. Z. S. Tao; B. L. Bai; X. W. He; W. Liu; H. Li; Q. Zhou; T. Sun; Z. L. Huang; K. K. Tu; Y. X. LV; W. Cui; L. Yang; Med. Biol. Eng. Comput., 2016, 54(12), 19591968.

46. Y. Liang; H. Li; J. Xu; X. Li; M. Qi; M. Hu; Int. J. Mol. Sci., 2014, 15(6), $9952-$ 9962.

47. S. Galli; M. Stocchero; M. Andersson; J. Karlsson; W. He; T. Lilin; A. Wennerberg; R. Jimbo; Osteoporos. Int., 2017, 28(7), 2195-2205.

48. S. Rapuntean; P.T. Frangopol; I. Hodisan; Gh. Tomoaia; D. Oltean-Dan; A. Mocanu; C. Prejmerean; O. Soritau; L.Z. Racz; M. Tomoaia-Cotisel; Rev Chim (Bucharest), 2018, 69(12), 3537-3544.

49. C. Garbo; J. Locs; M. D'Este; G. Demazeau; A. Mocanu; C. Roman; O. Horovitz; M. Tomoaia-Cotisel; Int. J. Nanomed., 2020, 15, 1037-1058.

50. P. T. Frangopol; A. Mocanu; V. Almasan; C. Garbo; R. Balint; G. Borodi; I. Bratu; O. Horovitz; M. Tomoaia-Cotisel; Rev. Roum. Chim., 2016, 61(4-5), 337344.

51. A. Mocanu; O. Cadar; P. T. Frangopol; I. Petean; Gh. Tomoaia; G. A. Paltinean; Cs. P. Racz; O. Horovitz; M. Tomoaia-Cotisel; R. Soc. Open. Sci., 2021, 8(1), 201785.

52. J. Y. Wang; Y. C. Liu; G. S. Lin; H. H. Chang; Y. T. Li; Y. C. Yang; H. Matsuyama; B. S. Lee; Y. W. Chen; K. L. Tung; Surf. Coat. Technol., 2020, 386, 125452. 
